\title{
An Adaptive Multimedia Messaging Service Framework for Mobile Telemedicine System
}

\author{
http://dx.doi.org/10.3991/ijim.v9i4.4627 \\ Andik Setyono \\ Dian Nuswantoro University, Jawa Tengah, Indonesia
}

\begin{abstract}
This paper presents an adaptive MMS framework to build a mobile telemedicine system for sending multimedia messages and for streaming audio and video files. With the proposed framework, the capability of MMS can be increased to send and receive multimedia files of larger sizes without degrading the quality of data which is not possible with the present MMS system. This feature is particularly important for telemedicine environments as a good quality of multimedia data is required to accomplish accurate medical diagnosis. The experimental results demonstrate that the proposed framework outperforms the existing MMS system with regard to the file size that can be transmitted and to the quality of data. The modified MMS framework is adaptive in the sense that it allows transmission of different sizes and types of multimedia messages. This framework can form the basis for developing a practical mobile telemedicine system.
\end{abstract}

Index Terms-MMS; Mobile telemedicine system; Multimedia message; Streaming

\section{INTRODUCTION}

Multimedia messaging service (MMS) is a mobile message sending and receiving service system that can not only send the text messages but also can send image, audio, video messages or combination of them. The usage of MMS is likely to increase rapidly as the need of including multimedia contents in message delivery using mobile technology increases day by day. According to a recent research report, mobile device penetration (mobile phone) has already reached up to $80 \%$ of the people in the world [1]. In Asia, the largest mobile phone using countries are China (with 644.8 million), India (with 391.6 million) and Indonesia (with 144.6 million), respectively [1]. MMS was introduced with the idea of replacing SMS with the advantage of using it for sending multimedia messages. MMS has become popular because of reasons such as almost of all mobile phones have MMS feature, low bandwidth, cost and device. Now, MMS can interoperate between different mobile phones and cellular service providers.

Currently, the MMS technology is used not only to send greeting cards, short video, voice and melody, but is also used to develop systems that are needed for sending and receiving multimedia contents in a mobile environment. A few examples of such MMS based systems are: telemedicine [2-5], streaming [6-8], mobile-commerce [9] and elearning [10]. In this paper, we focus on the use of MMS technology to develop a mobile telemedicine system that adaptive and cost-effective.

Telemedicine field is one of the research areas of biomedical engineering that needs multidisciplinary technologies, such as electronics, computer, telecommunications, medical procedures and transfer protocol [11]. The telemedicine is considered to be part of telehealth, which relies on telecommunication systems to interact with and monitor patients at distance [12]. A telemedicine system serves as an effective tool to enhance the quality of health care service remotely [13]. It aims at providing expertbased medical care to any place where the health care is needed [141. Telemedicine system can be supported more and more progressively with expanding mobile technology.

There are several types of mobile telemedicine technologies: wireless local networks (WLANs), mobile ad hoc networks (MANETs), wireless personal/patient networks (WPANs), third generation (3G) cellular phone technologies, radio frequency identification (RFID) technologies and wireless sensor networks (WSNs) [13]. In this paper, we present the basic architecture of a mobile telemedicine system and show how an adaptive MMS framework for a mobile telemedicine system application can be developed using the proposed architecture. We also use the developed MMS technology to build a prototype model for a mobile telemedicine system. The goals of this research are: (i) to develop an improved MMS technology, (ii) to build a mobile telemedicine prototype using the improved MMS technology and (iii) to evaluate the performance of the proposed MMS framework for mobile telemedicine system. This system is expected to be more adaptive than the existing MMS framework.

The rest of the paper is organized as follows: section 2 discusses mobile telemedicine system. The proposed development of the MMS framework and its descriptions are presented in section 3. Implementation and performance evaluation details are presented in section 4 and finally conclusion and future work are given in section 5 .

\section{Mobile Telemedicine SyStem}

In this section, a review of existing MMS technologies, telemedicine systems in general and mobile telemedicine systems is presented. This review is useful to develop an improved MMS technology which is capable of overcoming the limitations with the existing technology. The main goal of this research work is to create an adaptive MMS framework for a mobile telemedicine system.

\section{A. Review of existing MMS technology}

Currently, MMS technology is used to develop some mobile telemedicine systems, such as transmission of electrocardiogram [2], MMS technology in the operation theatre [15], Mobile MMS in trauma orthopedic telediagnosis [16] and MMS for teleradiology [17]. In this research work, we intend to enhance the existing capability of MMS technology in multimedia messages delivery. We 
also intend to use the improved MMS in streaming applications for audio and video files and to build a mobile telemedicine system. It is known that telemedicine system needs good quality of data for medical diagnosis and the data files are usually of large file size. We know that the existing MMS has limitation with respect to the size of multimedia message files. We can only send multimedia messages up to a maximum of $300 \mathrm{~KB}$ [18]. We investigate new methods to overcome this limitation with respect to the size of the file without any degrading the quality of the data.

MMS technology can be used to send multimedia messages and streaming audio and video file [8], even to remote areas which do not have Internet connections. We can connect Internet network using GPRS or UMTS provided by cellular operator. Further, MMS is easy to be developed for specific applications such as mobile telemedicine system. The limitations of the existing MMS technology are: limited message size, relatively high cost, restricted formats of multimedia files and the limitations of some mobile devices. It is expected that MMS technology will expand in line with the development of mobile devices. In this paper, we present an improved streaming method for audio and video using MMS technology. The concept of using MMS in streaming for audio and video file is as shown in Figure 1 [18].

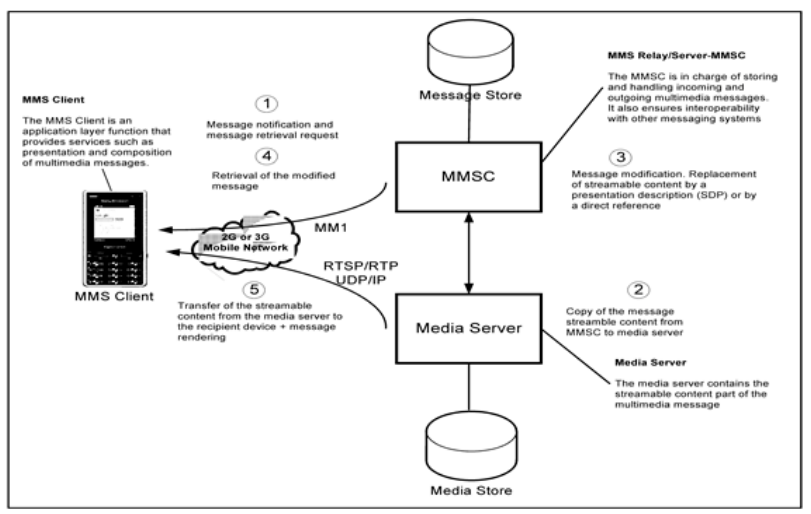

Figure 1. The Concept of Streaming Using MMS Technology [18]

\section{B. Study of telemedicine system}

Telemedicine is defined as the use of telecommunication to provide medical information and services remotely. In other words, telemedicine is the practice of health communication using audio, visual and data. Telemedicine offers benefits to patients, doctors and hospitals. Telemedicine provides direct benefits to the patients such as speeding up of a patient's access to referral centers, getting assistance while waiting for help from private doctors, and possibility of staying closer to the residence where family and friends can provide support directly.

The telemedicine system in general uses a high level technology and requires a huge amount of funding to provide for the necessary hardware and software, especially for the transmission of data using a high bandwidth channel. Basically the focus of telemedicine with ICT-based system is on the effectiveness, sustainability and acceptability [11]. This is an ordinary thing without considering about security, privacy and robustness because the technology is created to do it. How is about mobile phone that has small device, display, memory and low processing power such as MMS technology on mobile phone to send large file size? This is our focus research that is to enhance the MMS technology for transferring the large file size with preserve the quality of data. Besides, we also consider about security, privacy and robustness within developing system.

There are many challenges to overcome when implementing a telemedicine research project. Most notably, issues like recruitment, reporting ethical standards, confidentiality, licensing, perception and satisfactions must be considered so that the system can be made fast, robust and acceptable. Cost-effectiveness, safety and accuracy are additional factors to be considered.

\section{Study of the existing mobile telemedicine system}

Building a mobile telemedicine system application is not an easy task. Many aspects must be considered such as technique enablers, ethics, feasibility and usability. Mobile telemedicine is a field that has a vast potential to enhance accessibility of service, quality and continuity of care and significant cost savings in overall health care costs. But mobile telemedicine is still not well developed and is not widely socialized. This may be due to the fact that it needs an expensive technology and data transfer capabilities and also an approved data quality.

TABLE I.

COMPARISON OF TECHNOLOGIES FOR MOBILE TELEMEDICINE

\begin{tabular}{|c|c|c|c|}
\hline Technology & $\begin{array}{l}\text { Data } \\
\text { Rate }\end{array}$ & Frequency & $\begin{array}{l}\text { Max. } \\
\text { Range }\end{array}$ \\
\hline $\begin{array}{c}\text { IrDA } \\
\text { WLAN } \\
\text { IEEE 802.11a } \\
\text { IEEE 802.11b } \\
\text { IEEE 802.11g } \\
\text { MAN } \\
\text { IEEE 802.16 } \\
\text { IEEE 802.16a } \\
\text { Bluetooth IEEE 802.15 } \\
\text { Home RF } \\
\text { Mobile Technology } \\
\text { GSM } \\
\text { GPRS } \\
\text { EDGE } \\
\text { 3G / UMTS }\end{array}$ & $\begin{array}{c}54 \mathrm{Mbps} \\
11 \mathrm{Mbps} \\
54 \mathrm{Mbps} \\
<75 \mathrm{Mbps} \\
<75 \mathrm{Mbps} \\
723 \mathrm{kbps} \\
1 \mathrm{Mbps} \\
9.6 \mathrm{kbps} \\
171.2 \mathrm{kbps} \\
384 \mathrm{kbps} \\
2 \mathrm{Mbps}\end{array}$ & \begin{tabular}{|r}
$5.8 \mathrm{GHz}$ \\
$2.4 \mathrm{GHz}$ \\
$2.4 \mathrm{GHz}$ \\
\\
$10-66 \mathrm{GHz}$ \\
$2-11 \mathrm{GHz}$ \\
$2.4 \mathrm{GHz}$ \\
$2.4 \mathrm{GHz}$ \\
\\
$900 / 1800 / 1900 \mathrm{MH}$ \\
$\mathrm{z}$ \\
$900 / 1800 / 1900 \mathrm{MH}$ \\
$\mathrm{z}$ \\
$900 / 1800 / 1900 \mathrm{MH}$ \\
$\mathrm{z}$ \\
$1885-2200 \mathrm{MHz}$
\end{tabular} & $\begin{array}{r}5-10 \mathrm{~km} \\
10-100 \mathrm{~m} \\
<40 \mathrm{~m}\end{array}$ \\
\hline
\end{tabular}

Currently, wireless and cellular technologies are used for many purposes to build mobile telemedicine system. Table 1 shows a comparison of different technologies that are being used to build a mobile telemedicine system. In this research, we develop MMS application on mobile phone to process mobile telemedicine system. We also investigate new techniques for implementing an adaptive MMS framework for mobile telemedicine system.

\section{Proposed DEVElopment AND Discussion}

In this paper, we present the development of architecture, algorithm and application of MMS technology for an adaptive mobile telemedicine system that can be used for applications such as sending large multimedia files and streaming audio or video. The developed mobile telemedicine system consists of two parts, namely client and server applications. Both are integrated to form a complete mobile telemedicine system. We discuss about the proposed framework, algorithms and system modeling in the following sections. 


\section{A. Proposed framework}

In this research, we propose three basic architectures of MMS framework that are: (i) MMS framework for Internet applications (ii) MMS framework for mobile telemedicine system and (iii) MMS streaming for audio and video to support mobile telemedicine system. These three frameworks are integrated to develop a general mobile telemedicine system.

1) Proposed MMS framework for Internet applications

We propose an MMS framework for Internet applications. This framework is built as a client-server application as shown in Figure 2.

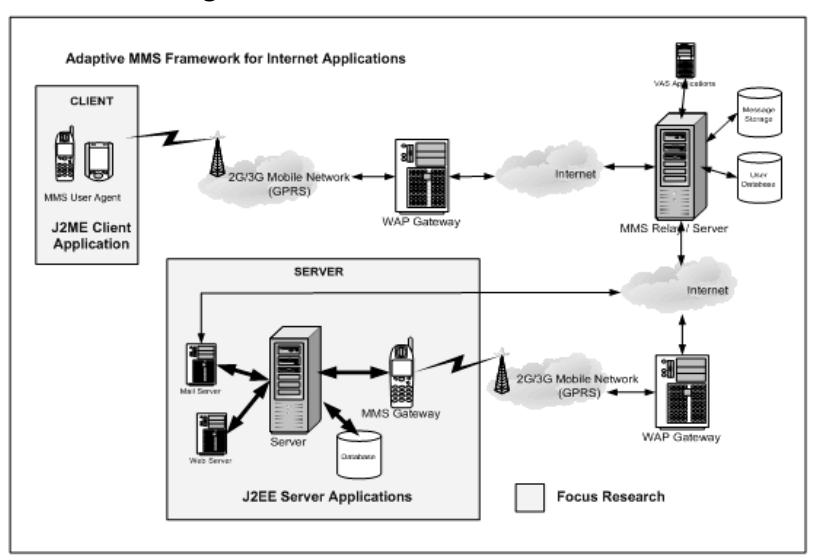

Figure 2. Proposed MMS Framework for Internet Applications.

MMS and telemedicine applications are developed on the client and server sides respectively. On the client side, we implement compression and split message techniques to send files of size larger than the permitted size. Messages that are sent to mobile phone recipient (MMS gateway) are retrieved by the database server, then merged and decompressed before storing. This architecture is useful not only for mobile telemedicine system, but also for other applications such as mobile commerce, mobile learning, mobile ticketing, etc. which use MMS technology and Internet. We can also add some techniques such as masking and cropping to get efficient and cost-effective for sending multimedia data using MMS technology.

2) Proposed MMS framework for mobile telemedicine

We develop an MMS framework to build an Internet based mobile telemedicine system. We propose MMS framework for mobile telemedicine system in this case is for diagnosis and consultation between nurse at patient home and doctor at the hospital. We develop MMS technology because it needs small device (mobile phone), low bandwidth (GPRS), large covered area and low-cost device and technology. This is suitable to access on remote areas and developed countries that still have high-cost Internet connection. Figure 3 shows the proposed MMS framework for mobile telemedicine system.

Multimedia message with a file size larger than the allowed size can be sent to mobile phone recipient (MMS gateway) using MMS technology. The compression and splitting techniques adopted at the client side ensure that the quality of data is not affected even if the file is of larger size [19]. This is important because the doctor at the hospital needs a good quality of data to accomplish the diagnosis process, so that the result will be more accurate.
The nurse at the patient's home sends multimedia message using MMS to the hospital, then the doctor can retrieve the data from the server using the telemedicine application on the server. After performing diagnosis, the doctor can input the diagnosis result into the system. The diagnosis result is automatically sent to the nurse at the patient's home by MMS. The nurse can also request for any additional information required using SMS. The server also responds to this request using SMS.

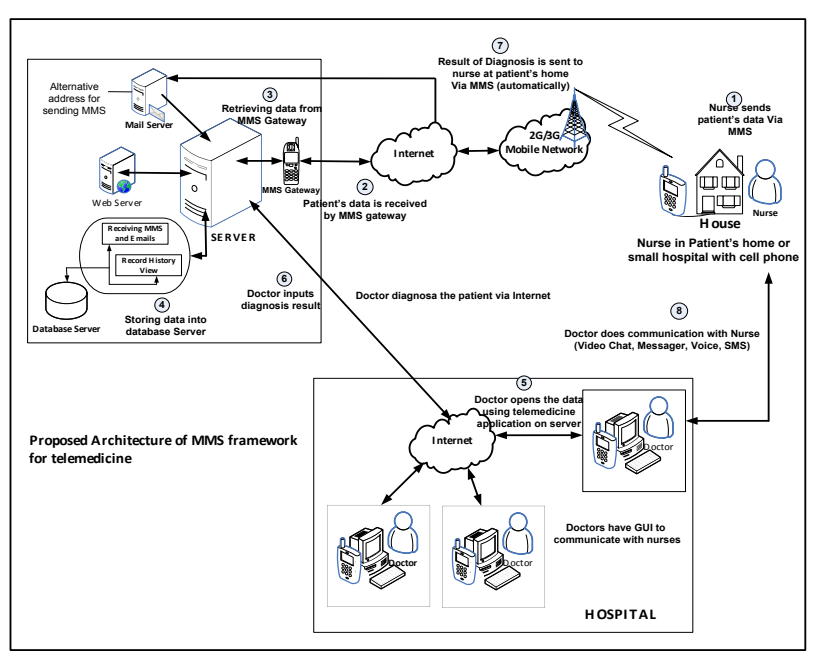

Figure 3. Proposed MMS Framework for Mobile Telemedicine System.

\section{3) Proposed MMS framework for audio and video} streaming

We develop a mobile telemedicine system using MMS streaming for audio and video. We discuss MMS in streaming because MMS technology can be used to do streaming specially for audio or video file. Figure 4 shows MMS framework for audio and video streaming. We use the streaming technique to send large audio and video files as an alternative for MMS.

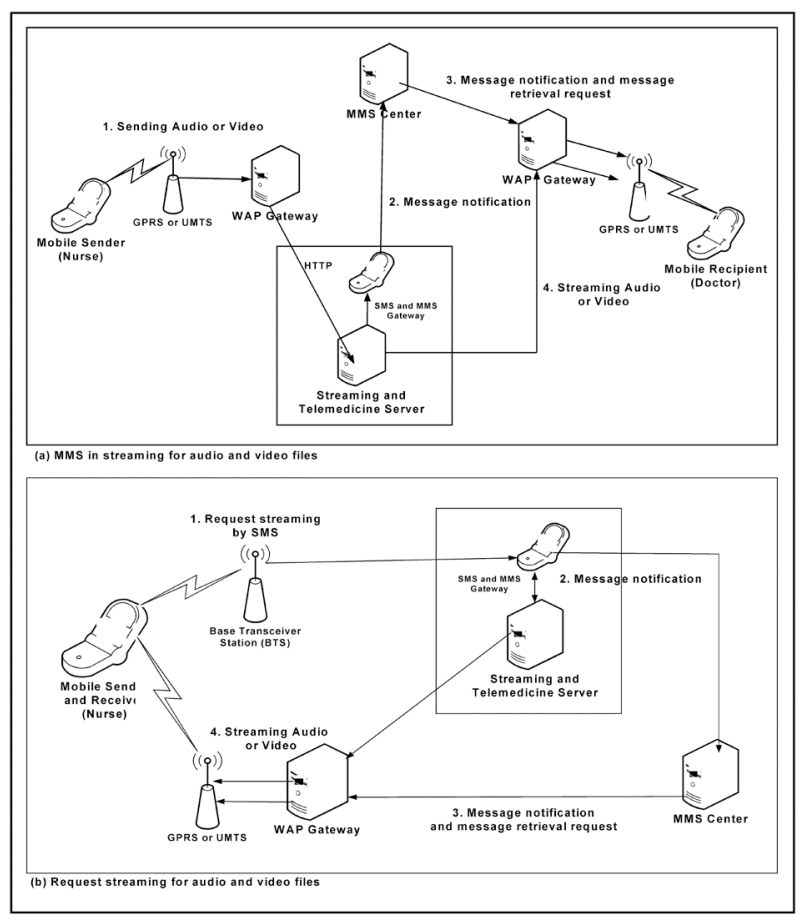

Figure 4. Proposed MMS Framework for Streaming Audio and Video. 
In Figure 4, we present the framework for audio and video streaming for mobile telemedicine system using MMS technology. Nurse at the patient's home can send the audio or video data to the server using HTTP protocol. The file uploading application of audio and video at the client (nurse) side includes the mobile phone number of the recipient (doctor). After receiving the data, the server sends the notification message to the MMS center which forwards this message along with a retrieval request message to the recipient. From the URL link address contained in the message, the doctor gets the audio and video data by streaming from the server. Nurse can also do the same by sending a request to the telemedicine server using SMS.

\section{B. Proposed algorithms}

\section{1) Algorithm for sending image message}

We present in this section, the MMS algorithms that are used to send and retrieve multimedia messages. Figure 5 shows the flow chart for sending large size images using compression, splitting and cropping techniques. The data compression process consists of three major steps which are removal of data redundancy, reduction in entropy and entropy encoding [20]. The splitting method is employed if the original file size $(O F S)$ is larger than the maximum capacity $(M C)$ permitted. If $M=O F S / M C$, the number of split files $N$ is given by [19]

$$
N= \begin{cases}M & \text { if } M \text { is an integer } \\ \text { Integer }(M+1) & \text { if } M \text { is a fraction }\end{cases}
$$

Let the original image be of size $(w x h)$ where $w$ and $h$ denote the width and height of the image, respectively. Then the size of each of the $N$ split images will be equal to $\{w \times(h / N)\}[19]$.

The algorithm of the splitting technique is shown in Figure 6.

\section{2) Algorithm for sending audio or video message}

We use Java technology to explain and simulate the algorithm for sending audio and video files. Java cannot be used to develop MMS application for sending audio and video files. This is because the Java library does not support the development of MMS application for sending audio and video files yet [22]. Java supports media player to play audio and video files using HTTP protocol. This problem can be solved by masking header method using image header (JPEG) to be known by MMS center.

a) Algorithm for downloading and storing the message

The split messages are received by the mobile phone recipient (MMS gateway), and then they are merged and decompressed into a single file before storing it into the database server. The algorithm for downloading and storing the message is shown in Figure 7.

Figure 7 explains the different processes that occur after the multimedia messages are received by the mobile phone recipient (MMS gateway). The OBEX downloader application [23] is modified to implement the retrieval, merging and decompression processes for the split files received from the MMS gateway. Finally, the file is stored into the database server and subsequently used for the telemedicine processes.

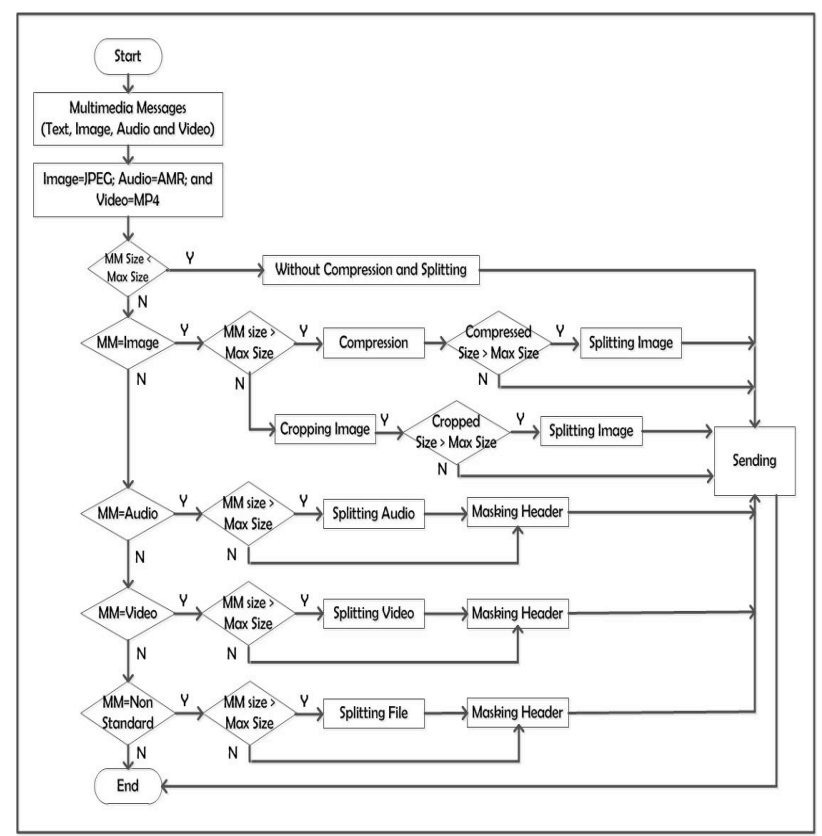

Figure 5. Development of Adaptive MMS Protocol.

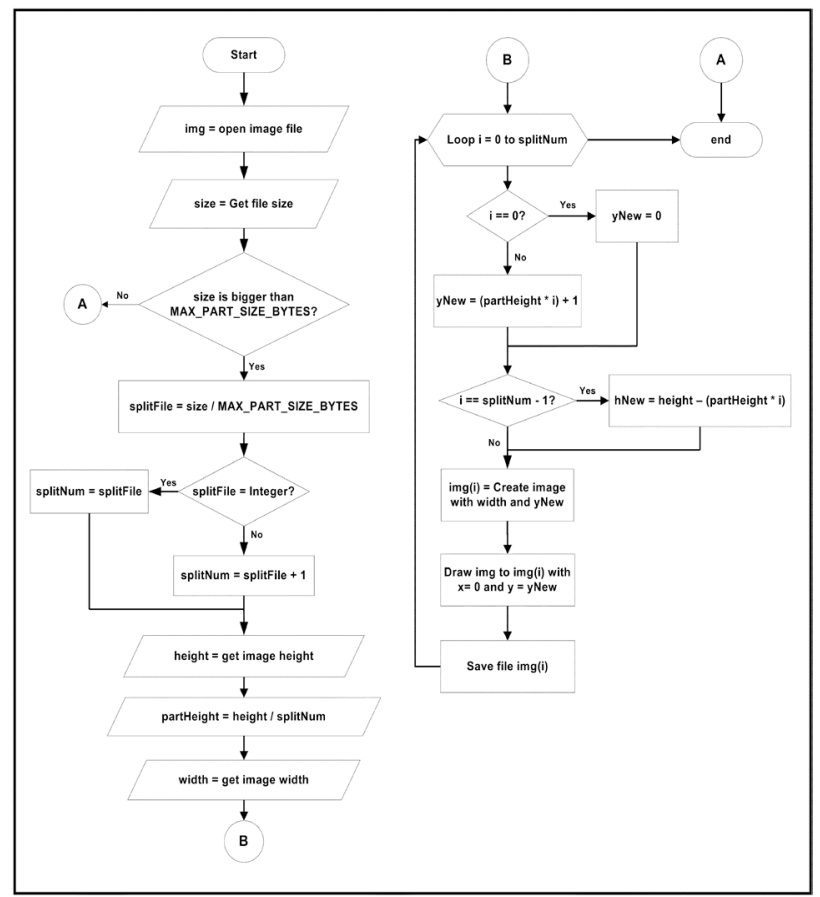

Figure 6. Algorithm of Splitting Message Technique Based on Pixel.

\section{3) Modeling of the Proposed system}

Use case modeling is used in requirements engineering for capturing the requirements of the system [21]. Figure 8 shows the use case diagram of the proposed mobile telemedicine system.

Nurse interacts with the system for sending data, requesting for patient history, uploading and streaming audio or video files and for receiving diagnosis results and consultation. The doctor can interact with the system for uploading and streaming audio or video files for the purposes of diagnosis and consultation. The administrator interacts with the system for billing and performing other administrative duties. 


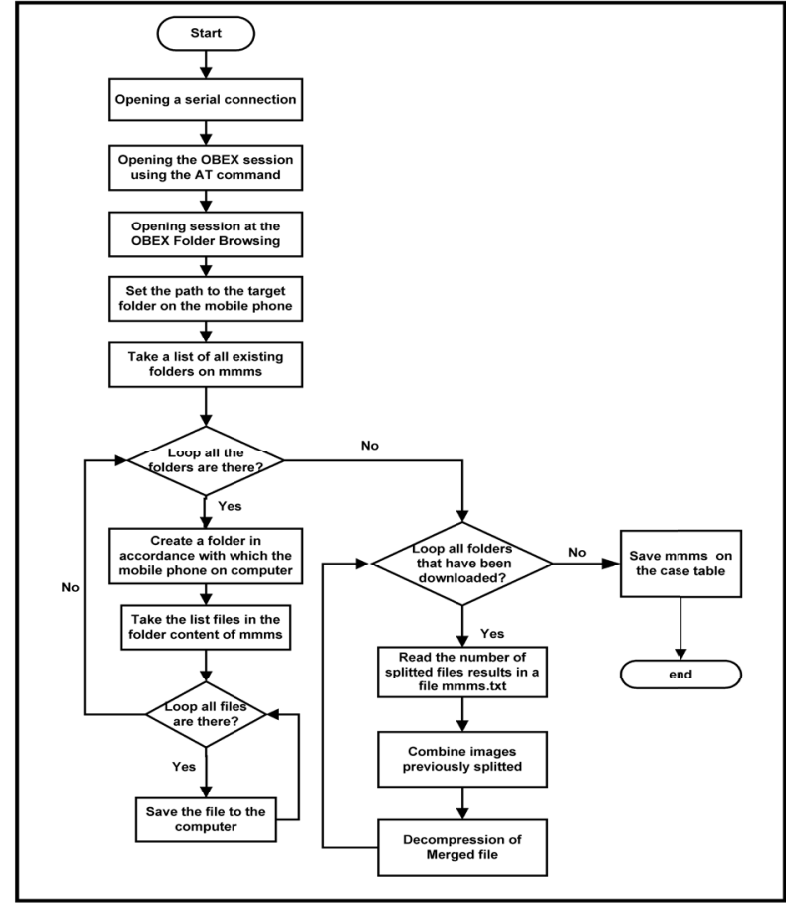

Figure 7. Proposed Algorithm for Downloading and Storing the Message.

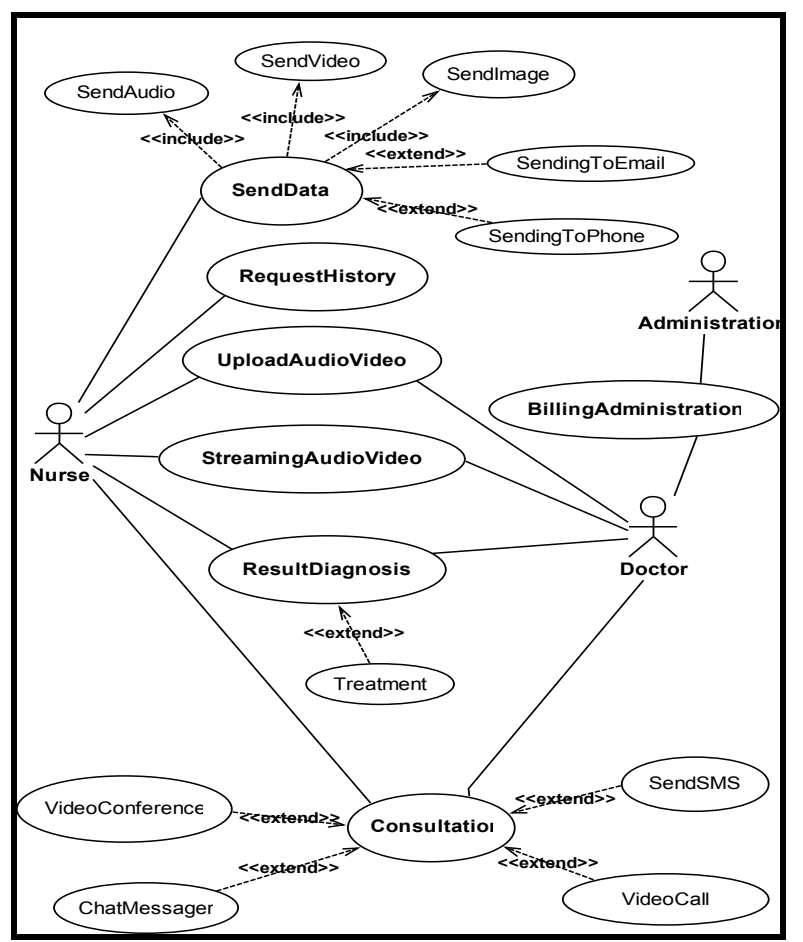

Figure 8. Use Case Diagram of the Proposed System.

\section{IMPLEMENTATION AND PERFORMANCE EVALUATION}

Mobile telemedicine system application is divided into two parts, namely client and server. On the client side, we develop MMS application to send multimedia messages and streaming audio and video files. While on the server side, we develop telemedicine system that can receive data from mobile phones in the form of multimedia messages. The implementation and evaluation details are discussed in the following sections.

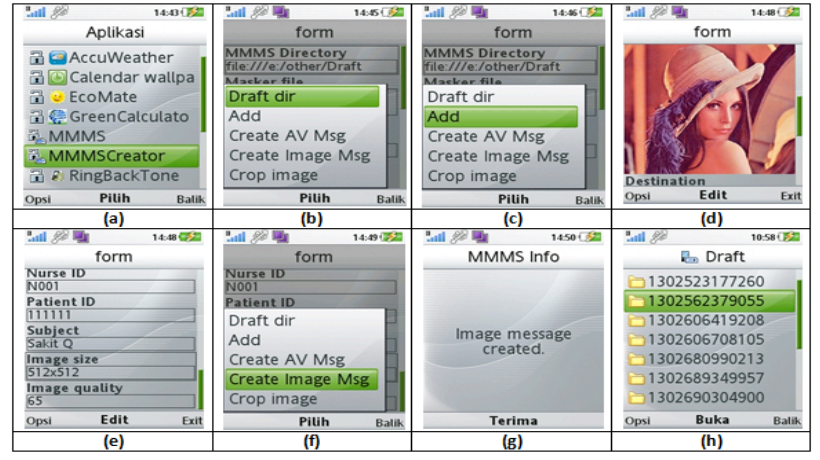

Figure 9. Development MMS Application to Create Message.

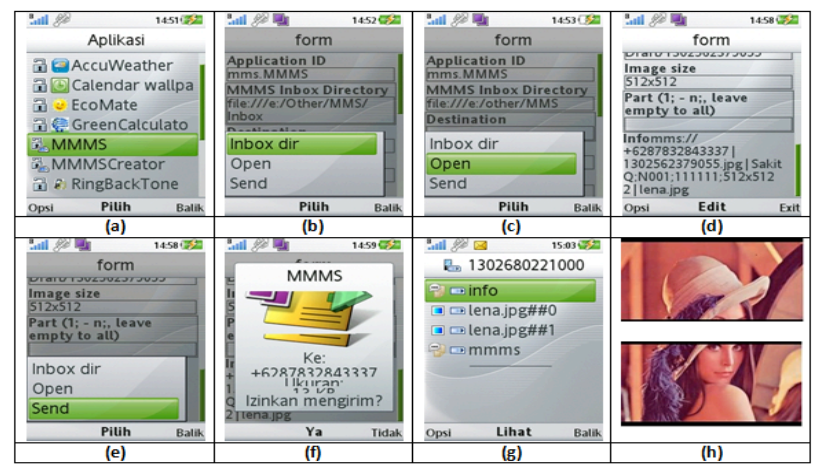

Figure 10. Development MMS Application to Send Message.

\section{A. Implementation}

The algorithms are developed using Java technology. Java 2 micro edition (J2ME) is used to develop the mobile applications on the mobile phone and Java 2 enterprise edition (J2EE) is used to develop the telemedicine applications on the computer server. To perform the simulation, we use Sony Ericsson mobile phone types J105i, J10i2 and K630i which have MMS and Java features.

\section{1) Mobile application}

MMS applications on the mobile phone comprise two components, namely, MMS application for sending files of size larger than the allowed one and MMS application for streaming audio and video files. Figure 9 and 10 illustrate the processes involved in sending large files containing multimedia messages.

We separate application of sending multimedia message to be two parts: (i) application for creating message and (ii) application for sending message. The types of message are made to be three: (i) message for containing image files, (ii) message for containing audio or video files and (iii) message for cropping the image file. We divide for sending the media because each message types have different algorithm. We add cropping image technique to solve if only a part of the image file needs for the next process. It will make size to be small so that it can be sent without compression and split technique. Then, the splitfiles which is sent to server side but there is the message failure sent whereas the message is part of split-files, to solve the problem, the server will give message notification using SMS to sender and request to send back the message containing part of file that is unsuccessfully sent, as in Figure 11(e). These strategies are to make costeffective within developing mobile telemedicine using MMS technology. 
In implementing the mobile telemedicine system, security issues need greater attention. This is because of the fact that this system is a client-server application, with the client on the mobile network and server on the internet network. The compression and splitting techniques are adopted for data security and for preservation of image quality. Data security is ensured since the delivered message is in the form of split files which are encoded. Image quality is preserved since no resizing of the image is done as in the conventional MMS method.

The MMS technology can be used not only for sending multimedia messages but also for streaming audio and video files. Figure 11 shows the layout of the MMS interface on the mobile phone for uploading the audio and video files.

Audio and video files are uploaded in the telemedicine server by the nurse at the patient's home by using HTTP protocol. On the server, there is an application to receive these audio and video files from the nurse. The telemedicine server in turn transmits these files to the recipient mobile phones (doctor and nurse) using streaming.

2) Server application

On the server, we have developed three applications which are described below.

(i) The first application is for downloading the split files from MMS gateway, merging them into a single file, decompressing and storing the file into the database server.

(ii) The second application is the telemedicine system (web-based) that processes the data sent from the nurse or doctor.

(iii) The third application is used for streaming the audio or video files to the recipient mobile phones (doctor or nurse).

The split files sent by the nurse are first received by the mobile phone MMS gateway. These split files are then downloaded, merged and decompressed before storing the message into the database server (MySQL). After the message is stored into the database, the doctor can use the telemedicine system to access the data, perform the diagnosis and input the diagnosis results back into the server. Figure 12 shows the interface of the download, merge and decompression.

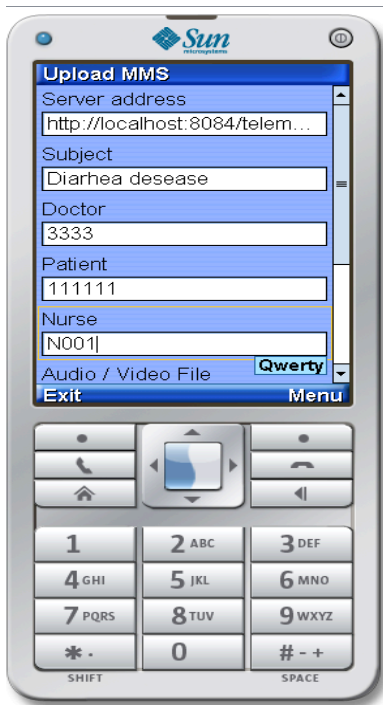

Figure 11. Interface of MMS Application for Uploading Audio or Video
The second application, namely, the telemedicine application, is a web-based application. The telemedicine application consists of several interfaces, such as main menu, input patient data, input nurse data, input doctor data, and input diagnosis result which are used by the telediagnosis and teleconsultation processes. The interface of the telemedicine application is shown in Figure 13.

The third application, namely, audio and video streaming is used to send message notification and to stream the data to the recipient (nurse and doctor). We make use of supporting software such as streaming server, apache and MySQL for developing the streaming application.

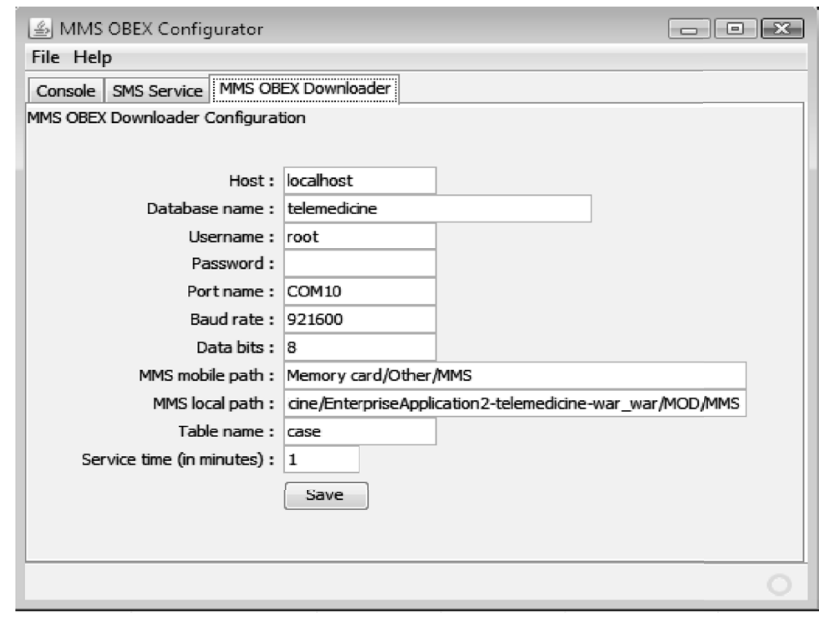

Figure 12. Interface of Download, Merge and Decompressed.

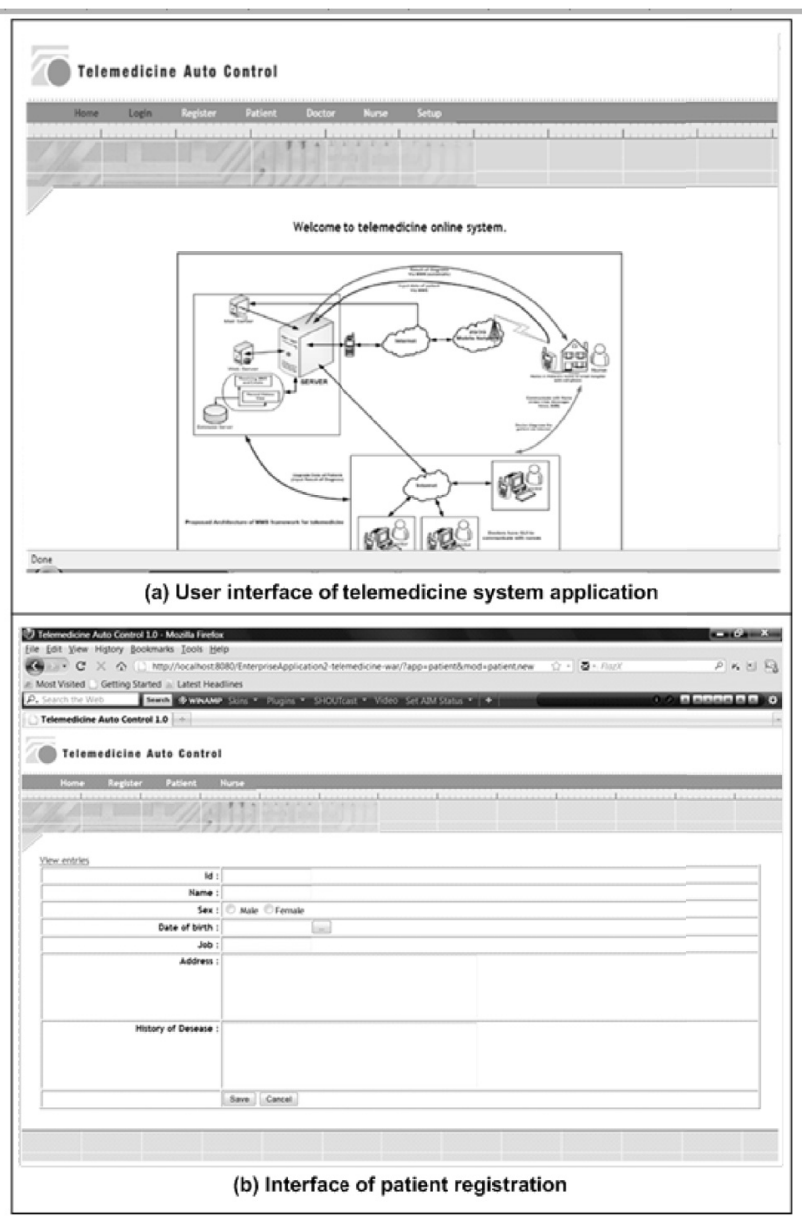

Figure 13. Interface of the Telemedicine Application on the Server. 


\section{B. Performance evaluation}

The proposed mobile telemedicine system has been implemented successfully and the experimental results show that the system can be used in a real telemedicine environment. To evaluate the performance of the system, we have performed a few experiments on the client and server sides. From the performance evaluation, it is expected that the system is able to provide an adaptive MMS framework for a mobile telemedicine system.

The simulated applications on the mobile phone for sending multimedia messages (JPEG images) have been tested and we have recorded several criteria, such as file size after compression and the number of files that are generated. We use the quality factor $50 \%$ in compression process. Table 2 shows the results of the performance measurement based on the compression and split techniques.

TABLE II.

MEASUREMENT PERFORMANCE BASED ON COMPRESSION AND SPLIT TECHNIQUES

\begin{tabular}{|c|c|c|c|}
\hline Data Size & $\begin{array}{c}\text { Reduced File } \\
\text { Size After } \\
\text { Compression }\end{array}$ & Split files & $\begin{array}{c}\text { Split File } \\
\text { Sizes }\end{array}$ \\
\hline $153 \mathrm{~KB}$ & $15 \mathrm{~KB}$ & 1 file & 15 \\
$115 \mathrm{~KB}$ & $33 \mathrm{~KB}$ & 2 files & 16,17 \\
$112 \mathrm{~KB}$ & $52 \mathrm{~KB}$ & 2 files & 25,27 \\
$101 \mathrm{~KB}$ & $12 \mathrm{~KB}$ & 1 file & 12 \\
$100 \mathrm{~KB}$ & $69 \mathrm{~KB}$ & 3 files & $20,26,23$ \\
$92 \mathrm{~KB}$ & $23 \mathrm{~KB}$ & 1 file & 23 \\
$66 \mathrm{~KB}$ & $19 \mathrm{~KB}$ & 1 file & 19 \\
$61 \mathrm{~KB}$ & $48 \mathrm{~KB}$ & 2 files & 24,25 \\
$55 \mathrm{~KB}$ & $14 \mathrm{~KB}$ & 1 file & 14 \\
$40 \mathrm{~KB}$ & $12 \mathrm{~KB}$ & 1 file & 12 \\
\hline
\end{tabular}

In sending multimedia files, we use the three algorithms described in section 3.2 for sending image files, for sending audio or video files and for streaming audio or video files. For text, it is not necessary to use the developed system because for the text file SMS technology is more efficient. To send image files, we use compression and split techniques before sending the files. To compress the image files, we use Huffman coding and we split the file based on the file size. To send audio and video files, we do not need to perform any compression again because the audio and video files are already compressed to the smallest size using $3 \mathrm{GP}$ and MP4 formats. In this case, we use only split and file masking techniques. The files are split based on the length of file and a header is included for each split file for identification by the MMS center. This procedure is required because Java library does not provide support for sending audio and video files. We mask each split file with the JPEG image format so that it will be identified as an image file by the MMS center. All the split files are merged into a single file and stored into the database server immediately after completing the downloading process. The third algorithm is used for sending audio or video files using streaming technique. In this work, we do not utilize the MMS streaming fully because we cannot modify the system on the MMS center. We upload audio and video files using HTTP technology along with the required identifiers in accordance with the database format. After the file is successfully stored into the database, the system gives a notification message to the recipient (doctor or nurse) to carry out streaming using the MMS technology.

The proposed system is still in the form of a prototype which has been developed using Java technology. The proposed mobile telemedicine system will yield the following benefits: (i) with the developed system, a patient can consult a doctor while living in a remote area, (ii) the doctor can diagnose the patient's information using a better quality of data without visiting the patient physically, (iii) the diagnostic results can be sent automatically by the system to the nurse at the patient's home through mobile phone number or email server based on the available communication system, and (iv) the system also provides streaming audio and video files using MMS technology. The nurse, who is at the patient's home, can communicate and consult with the doctor for further clarification using SMS, phone, or chat facilities of GPRS/UMTS.

\section{CONCLUSION AND FUTURE WORK}

This paper presents a study of an adaptive multimedia messaging service framework for mobile telemedicine system. The proposed framework is adaptive in the sense that it allows transmission of different sizes and types of files. We have investigated the development of MMS framework from the view point of enhancing the existing MMS limitations in message delivery and in streaming audio and video files. The simulation results show that the developed framework can be used successfully for sending larger size multimedia messages which is not possible by the existing MMS technology. In this paper, we have also presented MMS technology for streaming audio and video files. The aim of this paper is to explore the possibility of using MMS technology in developing a mobile telemedicine system and to the best of our knowledge this can be considered to be the pioneering work in this direction. The developed system is still in the form of a prototype. Research is under progress to optimize the proposed mobile telemedicine MMS framework using improved techniques and applications.

\section{REFERENCES}

[1] VietNamNet, Asia Mobile Phone Users. (2009, July 22) Vietnam $\begin{array}{lll}\text { Ranked 6th [online]. Available: } & \text {. }\end{array}$ http://www.myhphone.com/news/asia-mobile-phone-usersvietnam-ranked-6th.

[2] A. A. Tahat (2008) Mobile Personal Electrocardiogram Monitoring System and Transmission Using MMS, Proceedings of the 7th International Caribbean Conference on Devices, Circuits and Systems, April 28-30. http://dx.doi.org/10.1109/iccdes.2008.4542630

[3] W. Cheng, Y. Ming-Feng, C. Kuang-Chiung, and L. Ren-Guey (2007) Real-Time ECG Telemonitoring System design with Mobile Phone Platform. Elsevier, pp. 463-470, 2007.

[4] S. W. Ching, J. L. Hunang, and Y. C. Tong, Mobile Telemedicine Application and Technologies on GSM, IEEE.

[5] M. V. M. Figueredo, and J. S. Dias (2004) Mobile Telemedicine System for Home Care and Patient Monitoring. Proceeding of the 26th Annual International Conference of the IEEE EMBS. http://dx.doi.org/10.1109/iembs.2004.1403951

[6] G. Alireza, E. Tomoya, and T. Makoto (2008) A Multi-Source Streaming Model for Mobile Peer-to-Peer (P2P) Overlay Networks. Spriger-Verlag, pp. 122-131.

[7] S. T. Govind, S. Ganish, and G. Vishal (2010) Multimedia Streaming Technology 4G Mobile Communication Systems. International Journal on Computer Science and Engineering Vol. 02, No. 03. pp. 695-699, 2010.

[8] M. E. Mustafa (2005) Improved Implementation Solution and General Mobile Network Architecture for Interworking Between 
PAPER

\section{An Adaptive Multimedia Messaging Service Framework for Mobile Telemedicine System}

MMS and Streaming. International Journal of Communication Systems, pp. 335-357.

[9] Lei-da Chen and Gordon Skelton (2005) Mobile commerce development application, USA, Cybertech Publishing. http://dx.doi.org/10.4018/978-1-59140-806-2

[10] Kin Choong Yow and Sintiani Dewi Teddy (2008) MILCA - A Mobile and Interactive Learning Environment on Campus, Springer-Verlag Berlin Heidelberg.

[11] Y. S. Irawan, A. P. Koesoema, S. Soegijoko (2006) Sistem Telemedika Berbasis ICT Dalam Pengelolaan Masalah Kesehatan Masyarakat [ICT-Based Telemedicine System in Management of Public Health Problems]. Sekolah Teknik Elektro \& Informatika, Proceeding Konferensi Nasional Teknologi Informasi \& Komunikasi untuk Indonesia, pp. 223-226.

[12] A. Shaikh, M. Misbahuddin (2007) M. S. Memon, A System Design for a Telemedicine Health Care System.

[13] Y. Xiao, H. Chen (2008) Mobile Telemedicine, A Computing and Networking Perspective. Francis, An Auerbach Book, Chapter 18, pp. 349-363.

[14] C. S. Pattichis, E. Kyriacou, S. Voskaride, S. Pattichis, R. 1stepanian, C. N. Schizas (2002) Wireless Telemedicine Systems: An Overview. IEEE Antenna's and Propagation Magazine, Vol. 44, No. 2.

[15] A. Tewari, R. Oberoi, S. Garg, H. Kaur, D. Sood and S. Katyal (2008) Use of Multimedia Message Service Technology in the Operation Theatre: A Case Report. Indian Journal of Anaesthesia.

[16] T. A. Syed, Z. Sadiq, Y. R. Shah, M. Al-Saeed and M. H. Wetherill (2007) Role of mobile multimedia messaging service (MMS) in trauma and orthopedic telediagnosis. Springer-Verleg.
[17] N. Wai-Hoe, W. Ernest, and N. Ivan, Multimedia Messaging Service Teleradiology in the Provision of Emergency Neurosurgery Services, Jounal Surgical Neurology 67, pp. 338-341, 2006.

[18] Gwenaëlle Bodic, Mobile Messaging Technologies and Services SMS, EMS, MMS (2005) Second Edition, Chichester England, Jhon Wiley \& Sons.

[19] Andik Setyono, Md. Jahangir Alam and C. Eswaran (2010) Development and Implementation of Compression and Split Techniques for Multimedia Messaging Service Applications, ICINC.

[20] Tinku Acharya and Ping-Sing Tsai, JPEG2000 (2005) Standard for Image Compression: Concepts, Algorithms and VLSI Architectures, Canada, A Jhon Wiley \& Sons.

[21] Jim Arlow and Ila Neustadt(2005) UML 2 and the Unified Process second Edition Practical Object-Oriented Analysis and Design, Booch Jacobson Rumbaugh.

[22] Atanas Neshkov. (2009, June 17). MessagePart.java [online]. Available: http://members.fortunecity.com/neshkov/dj.html

[23] OBEX Commander. (2010, April 9) OBEX Commander for Win$\begin{array}{lll}\text { dows } & \text { Vista } & \text { Avline]. }\end{array}$ http://intradarma.com/OBEXCommander.html.

\section{AUTHOR}

Andik Setyono is with Dian Nuswantoro University, Jawa Tengah, Indonesia (setyonoandik@gmail.com).

Submitted 15 April 2015. Published as resubmitted by the author 20 August 2015. 